\title{
Microwave based Electrical Read-Out of Optical Sensors
}

\author{
Ulrich Nordmeyer ${ }^{1}$, Niels Neumann ${ }^{1}$, Xiaozhou Wang ${ }^{1}$, Torsten Thiel ${ }^{2}$, Konstantin Kojucharow ${ }^{3}$, Dirk \\ Plettemeier ${ }^{1}$ \\ ${ }^{1}$ Chair for RF and Photonics Engineering, TU Dresden, 01062 Dresden, Germany \\ ${ }^{2}$ AOS GmbH, Overbeckstr. 39a, 01139 Dresden \\ ${ }^{2}$ KMDC, Zur Bleiche 15, 01279 Dresden \\ ulrich.nordmeyer@tu-dresden.de
}

\begin{abstract}
Optical fiber sensors cover a wide range of applications. They offer versatile advantages including resilience against electromagnetic interference, biocompatibility and chemical resistivity. Even in environments with restricted accessibility, integration difficulties can be overcome by using Radio-overFiber (RoF) technology. Conventionally, the evaluation of optical fiber sensors is carried out optically by analysing the amplitude or spectrum of either the transmitted or the reflected light. A novel approach is to feed an RF modulated laser into the optical sensor and carry out a full electrical analysis of the resulting RF signal, which is changed by the sensors characteristics. This method will be investigated in the present paper along with different sensors and their applicability for this scheme will be discussed.
\end{abstract}

Keywords: optical fiber sensors, RF modulated laser, electrical analysis, wireless, radio-over-fiber

\section{Introduction}

Optical sensors are available for various quantities, e.g. temperature, strain, pressure, $\mathrm{pH}$, humidity and even electrical or magnetic field. Besides that, optical sensors offer some key advantages over competing sensing principles. Their main benefit is the resilience against electromagnetic interference. Furthermore, optical sensors provide immunity to corrosion, biocompatibility, high chemical resistivity and high sensitivity. Hence, they are suitable for measurement purposes in a wide range of applications, especially for those with harsh environmental conditions. These include the monitoring of buildings, healthcare, chemical processing and many more. [1, 2, 3] Different measuring methods based on optical sensors are well-known and share similar fundamental principles: The sensor, e.g. a fiber grating, is interfaced directly with fiber optics attached to a light source and the evaluation module. The light fed from the source into the optical sensor is evaluated after having passed the (passive) sensor. With photo detectors inside the evaluation module, the optical power, wavelength or phase of the transmitted or the reflected light may be measured.

While another key advantage of measurement setups based on optical sensors generally is the possibility of transmitting the signal over long distances without signal impairments, often times industrial environments do not allow to place the evaluation units remotely. In addition, setups with many sensors require many evaluation units which not only increases costs but also the complexity of cabling.

In environments with restricted accessibility or the need of many sensors, integration difficulties can be overcome by using Radioover-Fiber (RoF) technology [4]. In such an RoF setup each sensor is connected to a simple transducer that converts the signal from the optical to the electrical domain and transmits it wirelessly to a central station, which again carries out the signal evaluation for all sensors together. The optical-to-wireless converter basically consists of a photodiode, an amplifier and an antenna. For this principle to work along with such a simplified transducer, it is necessary to modulate the light source with an RF signal according to the desired carrier frequency of the radio transmission.

As explained above, conventionally the evaluation of optical fiber sensors is carried out optically by analysing the amplitude or spectrum of either the transmitted or the reflected light. Since the signal in RoF setups is RF modulated, an alternative and novel approach is to carry out a full electrical analysis of the received signal, which is changed by the sensor's characteristics. This method provides several benefits and is especially qualified for use in RoF applications since an RF modulation is present in those already, but can also be adapted to non-RoF applications by simply applying an RF modulation to the existing setup. 


\section{Sensors}

A typical application scenario for optical sensors is the measurement of temperature with fiber gratings. Their working principle is based on a periodic variation of the refractive index in the core of a single mode fiber. The grating acts as an interference filter with its specific resonance wavelength $\lambda_{R}$ according to

$$
\lambda_{\mathrm{R}}=\Lambda \Delta n_{\mathrm{eff}}
$$

with the grating period $\Lambda$ and the difference of the effective refractive indices of the involved modes $\Delta n_{\text {eff }}$ [5]. Fiber gratings having a period of some hundred nanometres belong to the subcategory of Fiber Bragg Gratings (FBG). The optical transmission characteristic of FBGs corresponds to a small band-stop filter around its resonance wavelength. Wider stop bands can be achieved by sweeping the grating period continuously from one end to another. Those gratings with a non-constant but consistently modulated grating period are called chirped FBGs. Depending on a change of temperature, the grating period increases or shrinks slightly. These changes again affect the resonance wavelength and the whole filter characteristic in form of a shift in wavelength. A measured change in the ratio of transmitted to inserted optical power of a stabilized laser source can be transferred back to a specific change in temperature provided that the filter characteristic is known. As controlling the temperature of the sensor leads to a more complex experimental setup, it will be kept constant while the shift of the filter characteristic relative to the inserted laser wavelength is simulated by changing the latter.

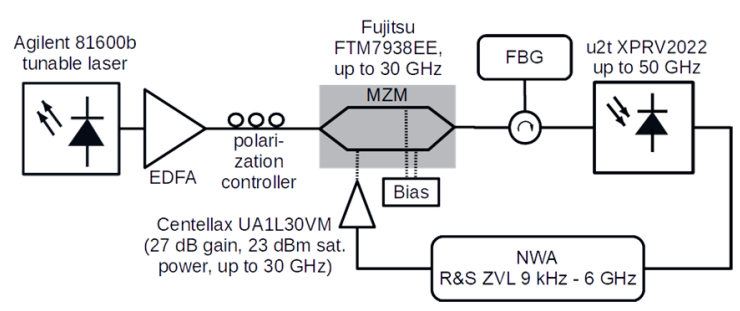

Figure 1: Schematic of the experimental setup

\section{Setup}

The approach of electrical read-out is tested with two different FBGs whereof one is chirped and the other one is not. Their characteristic parameters are shown in table 1 . The setup is identical for both sensors: A laser source with tuneable wavelength (TLS) is connected to an erbium-doped fiber amplifier (EDFA) to achieve sufficient optical power. The amplifier's output is connected to a Mach-Zehnder modulator (MZM) with a polarisation controller in between.
The first port of the sensor under test is connected to the output of the modulator, to the second port of the sensor a photodiode (PD) with sufficient RF bandwidth is connected. For the electrical read-out a 2-port Vector Network Analyser (VNA) is used whereas port one is connected to the MZM and port two to the PD. While the described setup integrates the sensor in transmission configuration, in the reflection variant a circulator is inserted between the MZM and the PD. One port of the sensor is connected to the circulator instead and the other one left open.

At first, the filter characteristics of both gratings have to be determined. By scanning the sensor with incident light in a wide but small stepped range of wavelengths, the sensor is analysed using an optical power meter. The resulting filter characteristic serves as reference for the outputs of the following electrical analysis.

The very same procedure is repeated by conducting full electrical S21 measurements on the VNA, that is amplitude and phase, in a frequency band from $9 \mathrm{kHz}$ to $6 \mathrm{GHz}$. The electrical analysis is performed for each wavelength the TLS is set to. Beforehand the whole setup is calibrated electrically by means of forward normalization at a wavelength with minimum attenuation. For the transmission configuration that is a wavelength beyond the filter notch, for reflection measurements a wavelength at the peak attenuation shown by the optical determined reference characteristic. This not only allows to calibrate the insertion loss of the whole setup including cables, electro-optical conversion, optical loss and opto-electrical conversion but also provides a reference of the group delay of the aforementioned setup. As a consequence, the measured S21 values are independent from the used electro-optical setup.

Table 1: Parameters of sensors under test

\begin{tabular}{|c|c|c|}
\hline \multicolumn{1}{|c|}{ Sensor } & Stop-band & Notes \\
\hline $\begin{array}{c}\text { FBG } \\
\text { L 25 mm }\end{array}$ & $\begin{array}{c}1537,7 \mathrm{~nm} \\
\pm 0,075 \mathrm{~nm}\end{array}$ & $\begin{array}{c}\text { apodized } \\
\text { cos-shape }\end{array}$ \\
\hline $\begin{array}{c}\text { Chirped FBG } \\
\text { L } ~ 5 \mathrm{~mm} \\
10 \mathrm{~nm} / \mathrm{cm}\end{array}$ & $\begin{array}{c}1536,9 \mathrm{~nm} \\
\pm 2,5 \mathrm{~nm}\end{array}$ & $\begin{array}{c}\text { apodized } \\
\text { tanh-shape }\end{array}$ \\
\hline
\end{tabular}

\section{Results}

For the transmission configuration, the measured electrical amplitude characteristics of the unchirped sensor are shown at selected wavelengths in Fig. 2. Those characteristics were acquired directly with the VNA in the form of S21 measurements. From these amplitude characteristics the filter spectrum of the sensor was calculated. 


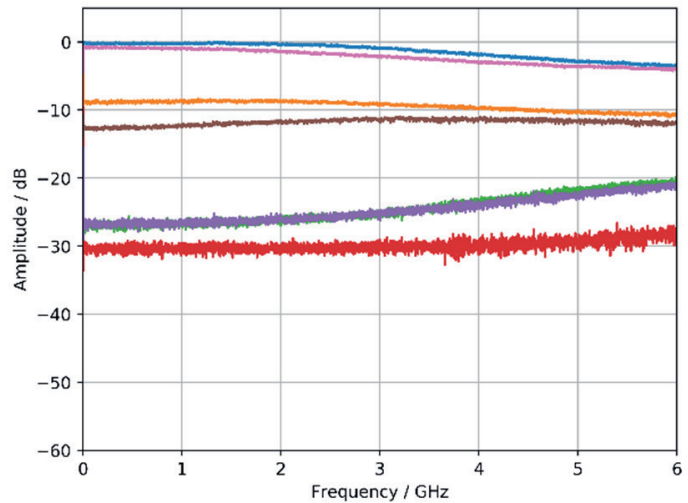

Figure 2: Electrical amplitude of $S 21$ measurements of unchirped FBG in transmission configuration

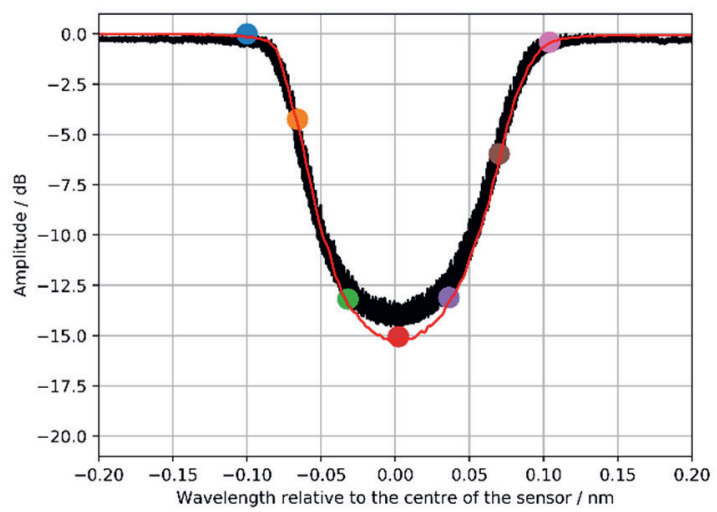

Figure 3: Electrically (red) vs. optically derived filter characteristic of unchirped sensor in transmission configuration

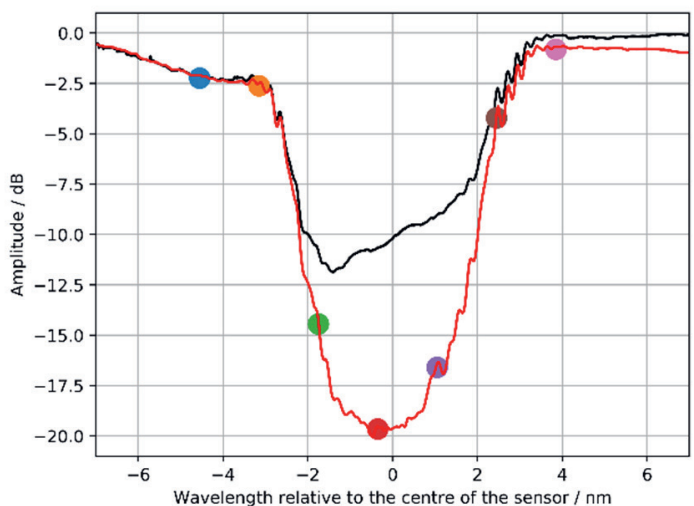

Figure 4: Electrically (red) vs. optically derived filter characteristic of chirped sensor in transmission configuration

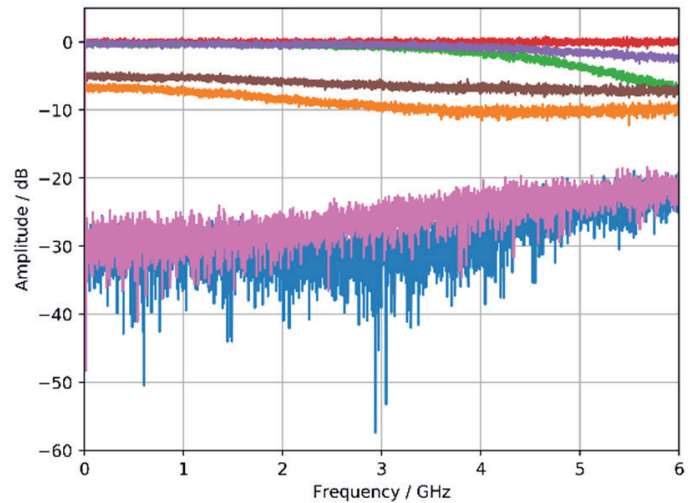

Figure 5: Electrical amplitude of S21 measurements of unchirped FBG in reflection configuration

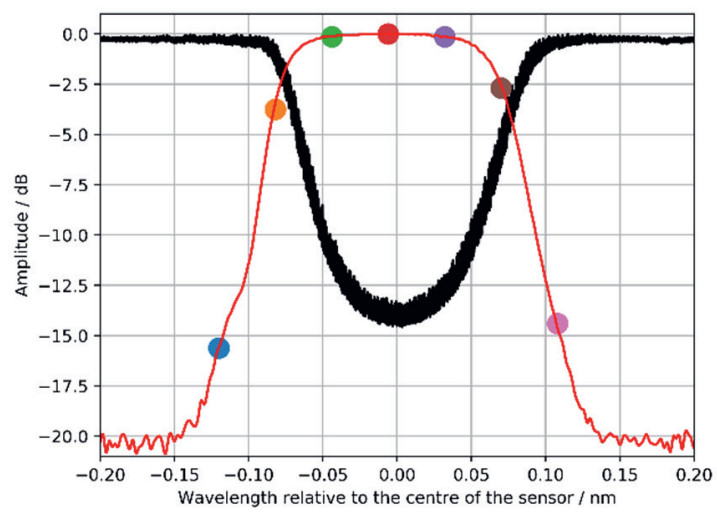

Figure 6: Electrically (red) derived filter characteristic of unchirped sensor in reflection configuration vs. optically measured characteristic in transmission configuration

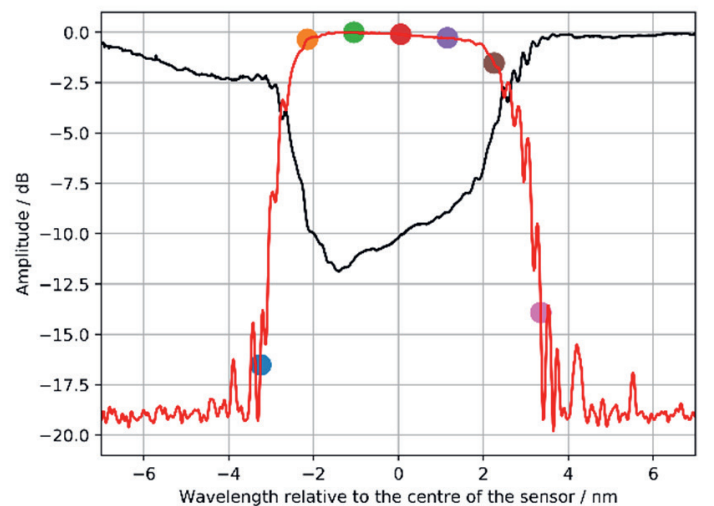

Figure 7: Electrically (red) derived filter characteristic of chirped sensor in reflection configuration vs. optically measured characteristic in transmission configuration 


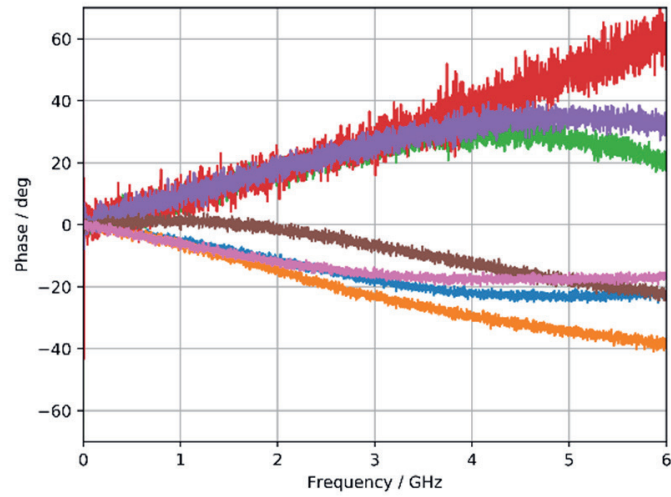

Figure 8: Electrical phase of S21 measurement of unchirped sensor in transmission configuration

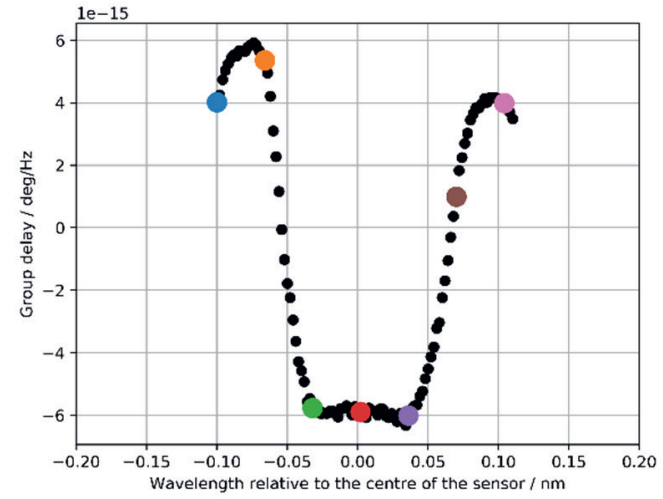

Figure 9: Group delay derived from el. Phase of unchirped sensor in transmission configuration

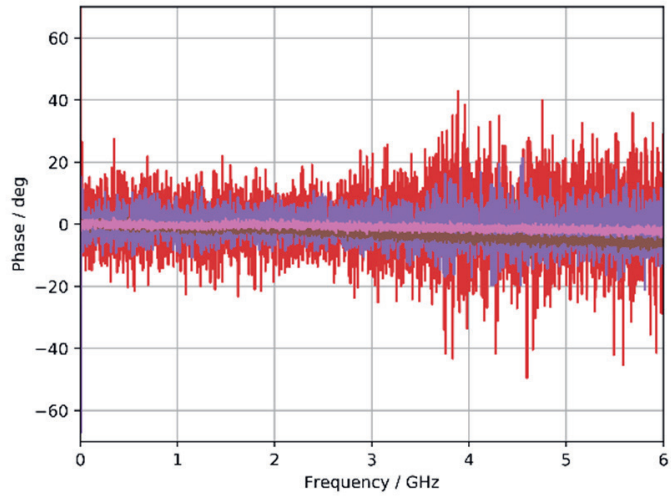

Figure 10: Electrical phase of S21 measurement of chirped sensor in transmission configuration

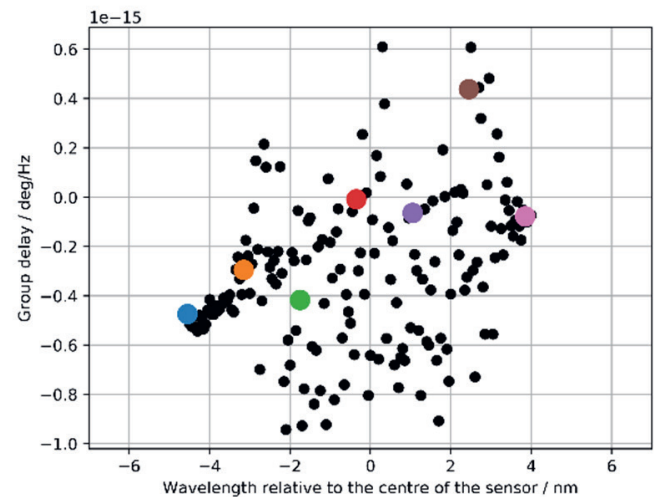

Figure 11: Group delay derived from el. Phase of chirped sensor in transmission configuration

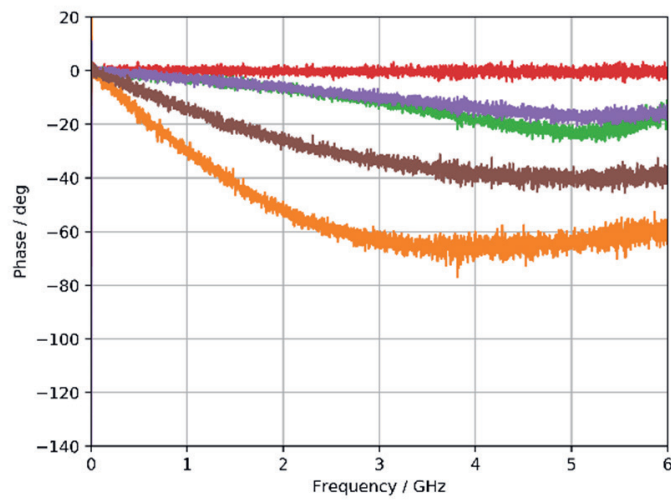

Figure 12: Electrical phase of S21 measurement of unchirped sensor in reflection configuration

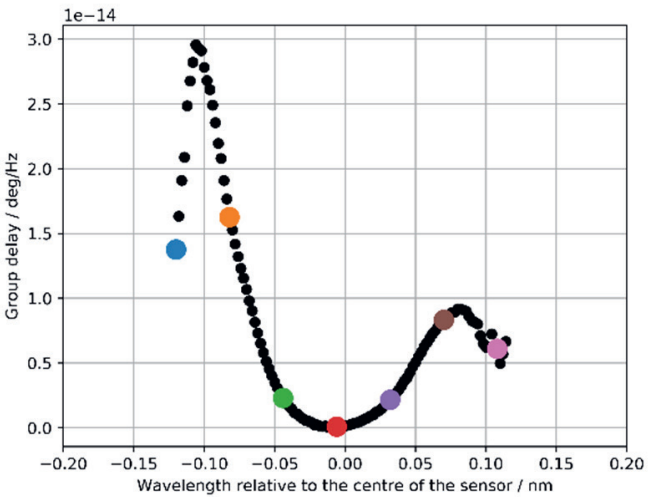

Figure 13: Group delay derived from el. Phase of unchirped sensor in reflection configuration

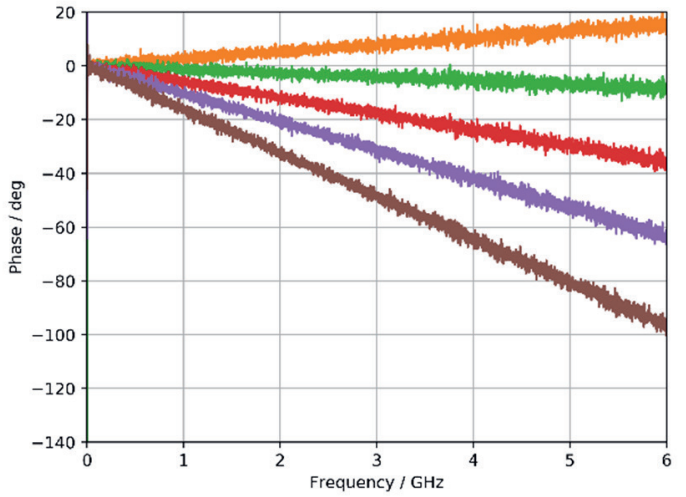

Figure 14: Electrical phase of S21 measurement of chirped sensor in reflection configuration

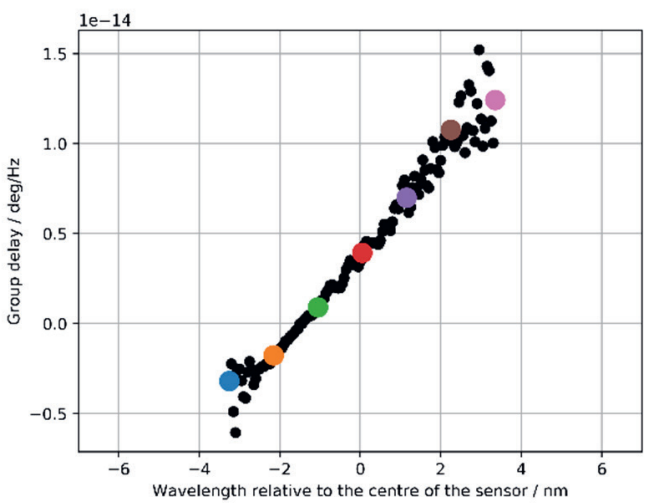

Figure 15: Group delay derived from el. Phase of chirped sensor in reflection configuration 
Figure 3 shows the back traced amplitude vs. wavelength curve in comparison to the reference acquired with the optical power meter. The calculation was implemented by taking the arithmetic mean of the measured amplitude in the frequency range of 0.5 to $2.0 \mathrm{GHz}$ for each measurement. To visualize the reconstruction better, Fig. 3 contains selected coloured dots that correspond to the depicted curves in Fig. 2. The same data is shown in Figs. 5 and 6 for the measurements in reflection configuration. It can be observed that the filter characteristic is inverted compared to the transmission setup.

Additionally the analogously back traced filter characteristics for the transmission and reflection measurements of the chirped sensor are shown in Figs. 4 and 7 . In comparison to the unchirped sensor, the filter characteristic spans over a wider range of wavelengths.

Because the S21 measurements performed with the VNA produce complex values for the amplitude, the phase information is included in the data. The derived phase characteristics are sloped according to the expected group delay variation. For all wavelengths outside of the stop-band in the transmission setup, the group delay equals the group delay of the optical fiber whereas within the stop-band it turns zero (ideally no transmission = photonic bandgap). This behaviour can be observed for both evaluated sensors in both setup configurations. The phase measurements are shown in Figs. 8 and 9 , the group delay characteristics in Figs. 10 and 11 . Again, the colours highlight the affiliation to the respective amplitude graphs. Based on the phase measurements the group delay was calculated according to

$$
\tau_{\mathrm{g}}=-\frac{\partial \Phi}{\partial \omega} .
$$

\section{Discussion}

The filter characteristics calculated from the electrical read-out show reasonable results compared to the conventionally determined graphs (Figs. 4-7). For the transmission experiment with the unchirped sensor, the electrically acquired curve has almost no deviation from the reference curve (Fig. 4). Highly remarkable is the much better resolution of the transmission filter characteristic of the chirped FBG (Fig. 6). In comparison to the conventional optical read-out, the new approach is affected less by the relative intensity noise (RIN) of the laser source, the ASE noise of the optical amplifier as well as by the noise currents in the photodiode, which leads to a better signal to noise ratio (SNR).
The stop-band attenuation of the grating is so high that even with the better sensitivity of the electrical measurement especially the phase measurements are still very noisy (Figs. 7 and 12). For the same reason the derived results for the group delay are unrepresentative. Besides, the positive slopes of some phase measurements and their corresponding negative values for the group delay that can be seen, may be attributed to the calibration procedure. A normalisation to the steepest positively sloped phase measurement would correct that phenomenon but in this paper all results are evaluated qualitatively only. In contrast to the transmission setup, the reflection configuration shows very smooth phase and group delay curves within the wavelength range of the stop bands. This matches with the previously explained observations: Because in transmission configuration the stop band attenuates the signal strongly, high noise ratios are resulting, while in reflection configuration the band-stop characteristic is flipped to a bandpass characteristic with accordingly low attenuation and therefore the noise contribution remains very low in the same band.

Another key property of the electrical evaluation can be studied in Figures 2 and 3 . While the positively sloped phase measurements are artefacts of the calibration process, the positive and negative slopes of the amplitude curves depend on another effect. The power of an optically modulated signal is split between the carrier and both sidebands. Considering the given measurement setup, the light of the TLS acts as the carrier while the sidebands are located symmetrically around it and their distance to the carrier is defined by the frequency of the RF-signal that is fed to the MZM. Higher modulation frequencies mean wider spectral separation of the sidebands. On the non-linear filter characteristic this can lead to different attenuations of each sideband. Considering the coloured markers in Figure 4 that correspond to the equally coloured amplitude graphs in Figure 2, that means e.g. for the measurement at the wavelength highlighted in orange: With increasing modulation frequency the sidebands move on the characteristic curve to each side of the carrier. The lower side band (LSB) moves to the right while the upper side band (USB) moves to the left. For small frequencies both sidebands remain in the almost linear slope of the curve and the lower attenuation of the USB compensates the higher attenuation of the LSB. So the impact of the rising frequency is negligible until one sideband leaves the linear range. Then the USB stays constantly at a low attenuation while the LSB is attenuated further 
until it reaches the peak attenuation. This leads to a negative slope which can be confirmed by Figure 2. For the measurement at the blue highlighted wavelength the effect is even stronger as the USB stays constantly at the lowest attenuation from the beginning. Since the photodiode only yields the mixing product of both sidebands with the carrier, the results for wavelengths on the right slope of the characteristic curve do not differ systematically from the measurements on the left slope, except from asymmetries immanent to the sensors filter curve. For all wavelengths near to the concave part of the filter curve, the presented effect produces a positive slope in amplitude. The same phenomenon can clearly be identified for the studies in reflection configuration (Fig. 3 vs. 5). This is an interesting property that on the one hand has to be taken into account when choosing the RF modulation frequency but can also be used for determining the spectral position of the edges of the gratingbased sensor characteristic.

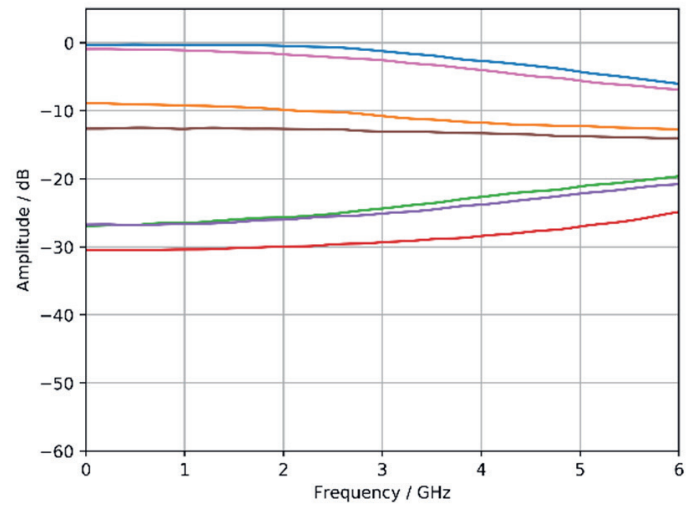

Figure 16: Analytically modelled amplitude for unchirped sensor in transmission configuration

To validate the stated theory, the electrical amplitude curves were analytically modelled according to the expected principle based on the measured filter characteristic. As it was calculated from a small band of low frequencies (equivalent to a DC measurement), it is not affected by the phenomenon beforehand. All calculations were performed using a modulation index of $m=1$ which equals a total power of $33 \%$ in both sidebands together. The results are depicted in Figure 16 and indicate the courses of curves as expected. Regarding higher frequencies the deviations from the measurements increase, but the synthetically generated data essentially matches the measured data. Further investigations should be carried out to identify the influence of different values for the modulation index and the non-constant group delay characteristic regarding the slopes of the amplitude measurements.

\section{Conclusion}

In this paper, the electrical read-out of optical sensors has been studied using Fiber Bragg Gratings and an RF-modulated laser source.

An introduction to optical sensors, their manifold measurement abilities and the conventional optical read-out has been given. Also, the context to Radio-over-Fiber applications has been pointed out where the novel approach originates from.

The results have shown new possibilities regarding the evaluation of optical sensors like the evaluation of the electrical phase and the group delay. In this context, the potentials and limitations of measurements in transmission versus reflection configuration have been discussed. Furthermore, it could be revealed, that the filter characteristic of an optical sensor does affect the shape of the measured electrical amplitude curves. This effect has to be taken into account when reconstructing filter characteristics by electrical read-out to avoid distortion as an impact of asymmetrical side band power distribution. Therefore, the precise reconstruction of filter characteristics from electrical S21 amplitude measurements should be conducted in small ranges of low frequencies only.

Future investigations will cover the influence of the modulation index and the non-constant group delay regarding the electrical read-out method.

\section{Acknowledgement}

This work has been funded by the EU and the Free State of Saxony in the project "OSMAT" within the framework of ESF-InnoTeam (SABNr. 100329864).

\section{References}

[1] Schuster, T.; Herschel, R.; Neumann, N. \& Schäffer, C. G. Miniaturized Long-Period Fiber Grating Assisted Surface Plasmon Resonance Sensor. Journal of Lightwave Technology, IEEE, 2012, 30, 1003-1008

[2] James, S. W., \& Tatam, R. P. (2003). Optical fibre long-period grating sensors: characteristics and application. Measurement science and technology, 14(5), R49.

[3] Neumann, N.; Schuster, T.; Häntzsche, E.; Nocke, A.; Plettemeier, D. Wireless Read-Out of Optical Fiber Sensors. Sensoren Und Messsysteme: Beiträge Der 18. ITG/GMAFachtagung 30. Mai - 01. Juni 2017 in Nürnberg. Berlin: VDE Verlag; 2017:197-201

[4] Neumann, N.; Schuster, T.; Plettemeier, D. Novel approach for simultaneous wireless transmission and evaluation of optical sensors. Photonics Asia, 2014

[5] Erdogan, T., Fiber grating spectra. Journal of Lightwave Technology 15(8), 1277-1294 (1997) 